\title{
NOUVELle
}

\section{Redéfinition du rôle de pTo au sein du pré-TCR}

\section{Les leçons de l'analyse moléculaire de pT $\alpha$ chez nos ancêtres}

Philippe Smelty, Céline Marchal, Thierry Jaffredo, Jean-Yves Sire, Julien S. Fellah
P. Smelty, C. Marchal, T. Jaffredo, J. S. Fellah : Université Pierre et Marie Curie, UMR-CNRS 7622, 9, quai Saint-Bernard, 75252 Paris Cedex 05, France.

jsfellah@snv.jussieu.fr

J.Y. Sire : Université Pierre et Marie Curie, UMR-CNRS 7138, 7, quai Saint-Bernard,

75252 Paris Cedex 05, France

\section{Maturation des lymphocytes T $\alpha \beta$ des mammifères}

L'efficacité du système immunitaire des mammifères est liée à la diversification du répertoire des lymphocytes $B$ et $T$ qui reconnaissent spécifiquement les antigènes présents dans l'organisme. Le répertoire des lymphocytes $T \alpha \beta$ est obtenu par le réarrangement des gènes codant les chaînes $\alpha$ et $\beta$ du récepteur à l'antigène (TCR, $T$ cell receptor) [1]. Ces événements de réarrangement se déroulent dans le thymus lors de la maturation des lymphocytes $T \alpha \beta$ à partir des cellules souches hématopoḯtiques provenant de la moelle osseuse. Une caractéristique essentielle de la genèse du TCR $\alpha \beta$ est le réarrangement séquentiel des locus TCRA et TCRB codant pour les deux chaînes de ce récepteur. À un stade précoce de la maturation des lymphocytes $T$ - stade appelé double négatif ou DN parce que les thymocytes n'expriment aucun des deux corécepteurs CD4 et $C D 8$ - le réarrangement du locus TCRB précède celui du locus TCRA. Les cellules ayant correctement réarrangé le locus TCRB expriment à leur surface une chaîne TCR $\beta$ fonctionnelle associée de façon covalente à une molécule invariante appelée la chaîne pré-TCR $\alpha$ (ou pT $\alpha$ ). L'expression à la surface des cellules DN du pré-TCR (TCR $\beta$-pT $\alpha$ ) associé aux molécules du complexe CD3 conditionne la suite de la différenciation des thymocytes en lymphocytes $T \alpha \beta$ matures (mécanisme désigné sous le terme de sélection $\beta$ ) [2]. La signalisation par le pré-TCR permet la survie et la proliféra- tion des cellules DN, l'exclusion allélique du second locus TCRB et la poursuite de la maturation des thymocytes notamment via le réarrangement du locus TCRA. Des expériences d'inhibition de l'expression de la pT $\alpha$ ont prouvé sa nécessité pour le développement normal des lymphocytes $T$. Ainsi, les souris dont le gène codant la pTa (PTCRA) a été invalidé possèdent dix fois moins de thymocytes qu'une souris normale et, en outre, ces thymocytes, incapables de franchir la sélection $\beta$, restent bloqués au stade DN [3].

Rôle essentiel de la chaîne pT $\alpha$ dans le développement des lymphocytes $T$

La PT $\alpha$ est une glycoprotéine membranaire de $33 \mathrm{kDa}$ possédant: une partie extracellulaire constituée d'un domaine d'immunoglobuline et d'un peptide de connexion contenant une cystéine qui permet son association à la chaîne TCR $\beta$; une région transmembranaire constituée d'acides aminés hydrophobes et une importante région intracytoplasmique, riche en proline, impliquée dans la transduction des signaux. Cette région intracytoplasmique, constituée de 30 acides aminés chez la souris et de 110 chez l'homme, semble également intervenir d'une part dans la rétention du préTCR dans le réticulum endoplasmique, et d'autre part dans l'internalisation et la dégradation de ce récepteur, permettant ainsi de limiter son expression à la surface cellulaire. En effet, une trop forte expression du pré-TCR à la surface des cellules pourrait engager la cellule dans un processus de tumorigenèse [4].
Les PTCRA humain et murin comportent quatre exons : l'exon 1 possède la région 5 ' non traduite et code pour le peptide signal et les premiers acides aminés de la région extracellulaire; l'exon 2 code pour le domaine d'immunoglobuline; l'exon 3 pour le peptide de connexion et l'exon 4 pour les régions transmembranaire et intracytoplasmique.

Aucun ligand du pré-TCR n'a été identifié à ce jour, et il était admis que la fonction du pré-TCR dépendait d'une homo-oligomérisation du récepteur impliquant des résidus chargés localisés dans le domaine extracellulaire de la pTa [5]. Récemment, le pré-TCR humain a été cristallisé et l'analyse de sa structure tridimensionnelle a montré que le domaine extracellulaire de la pT $\alpha$ interagissait non seulement avec le domaine constant de la chaîne TCR $\beta$ du pré-TCR mais également avec le domaine variable de la chaîne TCR $\beta$ d'un second pré-TCR entraînant ainsi la formation de dimères de pré-TCR à la surface des cellules [6]. Contrairement aux chaînes du TCR qui ont été identifiées dans toutes les lignées de vertébrés, la pT $\alpha$ n'avait été caractérisée que chez quelques espèces de mammifères (souris, homme, porc, vache) et sa présence chez le poulet, et de façon générale chez les vertébrés non mammaliens, avait été mise en doute. Cependant, le rôle important attribué à la pT $\alpha$ dans le développement des lymphocytes $T$, qui sont présents chez tous les vertébrés non mammaliens étudiés, plaidait en faveur de son existence chez ces derniers. Nous avons donc recherché PTCRA dans le 


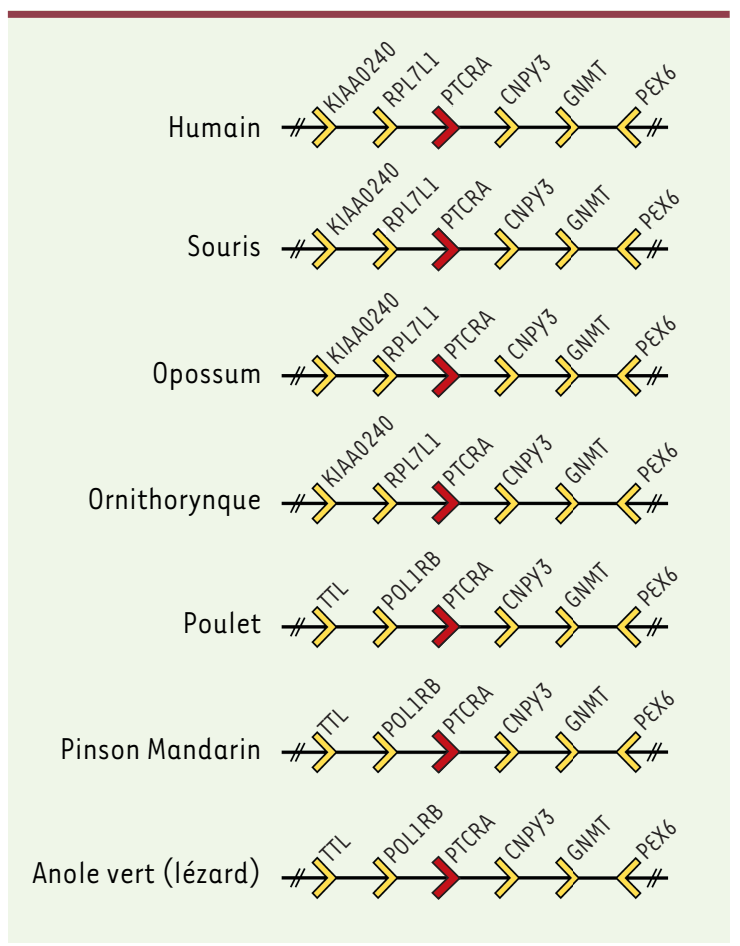

génome du poulet. Les méthodes directes de recherche in silico ayant échoué, nous avons procédé par synténie ${ }^{l}$ [11].

\section{Longueur du domaine}

intracytoplasmique de pT $\alpha$ et signalisation via le pré-TCR au cours de l'évolution

L'analyse des gènes associés au PTCRA chez quatre mammifères (souris, homme, porc, vache) montre une bonne conservation de la synténie avec, en particulier, les gènes RPL7L1 (ribosomal protein L7-like 1) et CNPY 3 (canopy 3) situés respectivement en amont et en aval de PTCRA (Figure 1). En nous appuyant sur l'étude de cette synténie, nous avons pu identifier PTCRA chez des représentants de toutes les lignées de mammifères, y compris les monotrèmes ${ }^{2}$ [7]. La synténie est moins bien conservée chez les sauropsidés qui regroupent les oiseaux et les reptiles actuels. En aval de PTCRA, la syn-

\footnotetext{
${ }^{1}$ Groupes de gènes dont le voisinage et l'organisation sont conservés sur plusieurs génomes. La synténie se caractérise par deux types de relations: de colocalisation (intragénome) ; ou de correspondance (intergénome) [http://www. ihes.fr/ carbone/M2BIM-sem6.pdf].

${ }^{2}$ Voir $[12,13]$ pour informations sur les évolutions génétiques des monotrèmes (ornithorynques, etc.).
}

Figure 1. Organisation des gènes autour de PTCRA (gène codant pour la pTo) dans le génome de quelques représentants de vertébrés. Chez les mammifères (homme, souris, opossum et ornithorynque), l'ordre des gènes (synténie) est conservé avec RPL7L1 et CNPY3 situés respectivement en amont et en aval de PTCRA. Chez les sauropsidés (oiseaux et lézards), la synténie est bien conservée en aval de PTCRA avec notamment CNPY3 et GNMT mais elle diffère de celle des mammifères en amont de PTCRA, où l'on trouve POLIRB et TTL à la place de RPL7LI et KIAAG240. Cette région génétique a probablement subi un réarrangement chromosomique au moment de la divergence entre les sauropsidés et les mammifères. GNMT : glycine $N$-methyltransferase ; KIAA0240 : pas de nom officiel ; PEX6 : peroxisomal biogenesis factor 6; POLR1B : polymerase (RNA) I polypeptide $B$; PTCRA : pre T-cell antigen receptor alpha ; RPL7L1 : ribosomal protein L7-like 1 ; $\Pi \mathrm{TL}$ : tubulin tyrosine ligase ; CNPY3 : canopy 3.

ténie est bien conservée avec notamment CNPY3, mais elle ne l'est plus en amont où l'on trouve le gène POLIRB (polymerase [RNA] I polypeptide B) à la place de RPL7L1. En utilisant CNPY3 comme cible dans les banques de données, nous avons pu identifier chez divers sauropsidés (3 oiseaux et un lézard) les régions génomiques contenant CNPY3 puis, par analyse des séquences en amont de ce gène, nous avons trouvé PTCRA chez ces espèces [7]. L'analyse de la structure génique de PTCRA chez ces sauropsidés montre que les quatre exons sont conservés chez les amniotes ${ }^{3}$ [7]. Cependant, la comparaison des séquences de pT $\alpha$ chez les mammifères et les sauropsidés a révélé des différences qui remettent en question certaines interprétations de la structure et de la fonction de cette protéine. La première particularité est l'absence, chez les sauropsidés et chez certains mammifères, d'une importante région intracytoplasmique [7]. Chez les oiseaux et le lézard, cette région n'est constituée que de sept acides aminés et ne contient pas de proline. Chez certains mammifères comme les lagomorphes (lapin, pika), les marsupiaux (opposum, wallaby) et les monotrèmes (ornithorynque), elle n'est constituée

\footnotetext{
${ }^{3}$ Les amniotes sont des vertébrés tétrapodes qui possèdent un amnios, ou sac amniotique, protégeant l'embryon ou le fœtus. Les reptiles, les oiseaux et les mammifères sont des vertébrés amniotes.
}

que de 6 à 22 acides aminés et contient peu ou pas de proline. Chez ces espèces, un long domaine intracytoplasmique ne semble donc pas être requis pour le développement des lymphocytes T et sa courte taille n'aurait pas de conséquences sur les mécanismes responsables de la diversification du répertoire T. Ces données semblent être en contradiction avec celles obtenues chez les souris transgéniques possédant une pT $\alpha$ dépourvue de région intracytoplasmique ou de motifs riches en proline : chez ces souris la signalisation en aval du pré-TCR est fortement inhibée, entraînant une nette diminution du nombre de thymocytes et un défaut dans le processus de la sélection $\beta$ [8]. Les données que nous avons obtenues indiqueraient que, chez les espèces qui ont une chaîne pT $\alpha$ dépourvue de véritable domaine intracytoplasmique, la signalisation relayée par le pré-TCR est assurée exclusivement par le complexe CD3 associé à ce récepteur (Figure 2).

Chez tous les primates, la présence d'une pT $\alpha$ prolongée par un long domaine intracytoplasmique, qui permet une mobilisation de molécules de signalisation impliquant les motifs riches en proline, pourrait être considérée comme un avantage évolutif [9]. Le long domaine intracytoplasmique permettrait d'optimiser la signalisation via le pré-TCR, compensant ainsi la faible expression de ce dernier à la surface des thymocytes immatures. Des expériences de mutagenèse avaient permis d'identifier dans le domaine extra- 


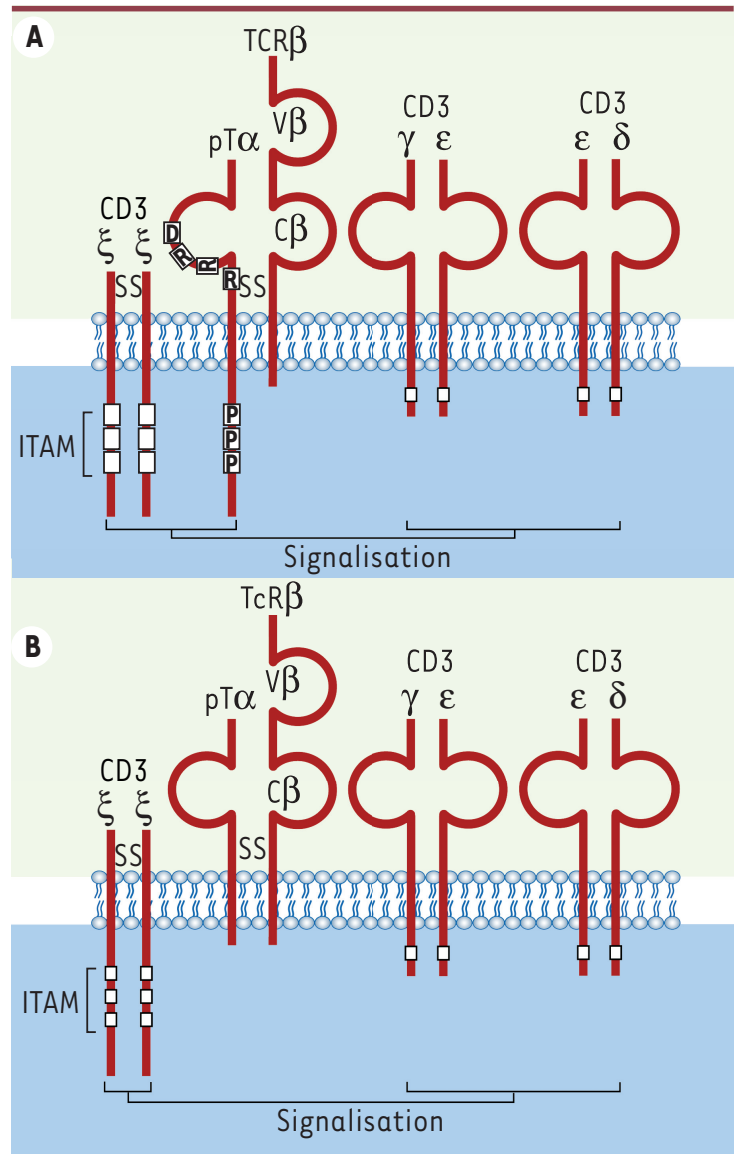

cellulaire des pT $\alpha$ murine et humaine plusieurs résidus chargés (acide aspartique et arginine) jouant un rôle crucial dans I'homo-oligomérisation du pré-TCR nécessaire à sa fonction [10]. La substitution d'un de ces résidus par un acide aminé différent annihile la signalisation via le pré-TCR. Or, nous montrons que ces résidus ne sont pas conservés dans la pT $\alpha$ de la plupart des espèces de mammifères et chez les sauropsidés: ils sont le plus souvent remplacés par des acides aminés polaires ou non chargés sans que cela semble avoir de conséquences sur le développement des lymphocytes T [7].

\section{Conclusion}

Cette étude comparative de la pT $\alpha$ nous a permis de montrer que cette molécule, cruciale pour le développement des lymphocytes $T$, était déjà présente chez l'ancêtre des vertébrés amniotes il y a plus de 310 millions d'années. Nos analyses ont montré que la plupart des pT $\alpha$ ne possédaient pas le domaine intracy-

Figure 2. Représentation schématique du complexe pré-TCR à la surface des thymocytes précoces. $A$. Chez l'homme et la souris, la signalisation via le pré-TCR implique les séquences riches en proline de la région intracytoplasmique de la pT $\alpha$ et les motifs ITAM (immunoreceptor tyrosine-based activation motif) des molécules du complexe CD3. Les résidus acide aspartique (D) et arginine (R) présents dans le domaine extracellulaire de la pT $\alpha$ sont impliqués dans l'homo-oligomérisation du pré-TCR. B. Chez certains mammifères (lagomorphes, marsupiaux et monotrèmes) et chez les sauropsidés, la région intracytoplasmique de la pT $\alpha$ est extrêmement réduite et ne comporte pas de proline ( $P$, voir $A)$, de même les résidus $D$ et $R$ ne sont pas conservés dans le domaine extracellulaire, indiquant que, chez ces espèces, la pT $\alpha$ aurait un rôle négligeable dans la signalisation via le pré-TCR.

toplasmique ni les résidus extracellulaires identifiés chez la souris et l'homme comme étant importants pour la signalisation du pré-TCR. Elles indiquent que ces différences n'ont aucune incidence sur la production des lymphocytes $T$ et conduisent à une redéfinition de la véritable fonction de la pT $\alpha$ au sein du pré-TCR. Cette fonction serait uniquement de permettre, à un stade où la chaîne TCR $\alpha$ ne peut s'exprimer, l'expression à la membrane d'une chaîne TCR $\beta$ fonctionnelle afin de recruter les molécules du complexe CD3. La pT $\alpha$ ne serait donc pas impliquée dans la signalisation chez la plupart des amniotes. Le long domaine intracytoplasmique riche en proline apparu à la suite de mutations de la région carboxy-terminale au cours de l'évolution des mammifères placentaires aurait été sélectionné dans différentes lignées (rongeurs, primates, etc.) car il permettrait une activation plus efficace des voies de signalisation ainsi qu'une régulation plus fine de l'adressage du pré-TCR à la membrane plasmique. $\diamond$

Reappraisal of the role of pT $\alpha$ for preTCR signaling: lessons from non mammalian vertebrates

\section{CONFLIT D'INTÉRÊTS}

Les auteurs déclarent n'avoir aucun conflit d'intérêts concernant les données publiées dans cet article.

\section{REMERCIEMENTS}

Les auteurs remercient Sophie Gournet pour les illustrations. Philippe Smelty a bénéficié d'un financement de la Société Française d'hématologie.

\section{RéFÉRENCES}

1. Fowlkes BJ, Pardoll DM. Molecular and cellular events of T-cell development. Adv Immunol 1989 ; 44 : 207 64.

2. Fehling HJ, Krotkova A, Saint-Ruf C, von Boehmer H. Crucial role of the pre-T-cell receptor alpha gene in development of alpha beta but not gamma delta T cells. Nature $1995 ; 375: 795-8$.

3. von Boehmer H. Unique features of the pre-T-cell receptor alpha chain : not just a surrogate chain. Nat Rev Immunol 2005 ; 5 : 571-7.

4. Yamasaki S, Saito T. Molecular basis for the pre-TCRmediated autonomous signaling. Trends Immunol $2007 ; 28: 39-43$.

5. Pang SS, Berry R, Chen Z, et al. The structural basis for autonomous dimerization of the vpre-T-cell antigen. Nature $2010 ; 467: 844-8$.

6. Bellavia D, Campese AF, Checquolo S, et al. Combined expression of pT $\alpha$ and Notch 3 in T cell leukemia identifies the requirement of pre TCR for leukemogenesis. Proc Natl Acad Sci USA 2002 ; 99 : 3788-93.

7. Smelty P, Marchal C, Renard R, Sinzelle L, et al. Identification of the pre-T-cell receptor alpha chain in nonmammalian vertebrates challenges the structure-function of the molecule. Proc Natl Acad Sci USA 2010 ; 107 : 19991-6.

8. Aifantis I, Borowski C, Gounari F, et al. A critical role for the cytoplasmic tail of pT $\alpha$ in the lymphocyte development. Nature $2002 ; 3: 483-8$.

9. Navarro MN, Nusspaumer $G$, Fuentes $P$, et al. Identification of CMS as a cytosolic adaptator of the human pT $\alpha$ chain involved in pre-TCR function. Blood $2007 ; 110: 4331-340$.

10. Yamasaki $S$, Ishikawa $\varepsilon$, Sakuma $M$, et al. Mechanistic basis of pre-T-cell receptor-mediated auronomous signaling critical for thymocyte develpment. Nat Immunol $2006 ; 7: 67-75$.

11. Médigue C, Bocs S, Labarre L, et al. L'annotation in silico des séquences génomiques. Med Sci (Paris) $2002 ; 18: 237-50$.

12. Gilgenkrantz $\mathrm{S}$. Le génome de l'ornithorynque : à la croisée des chemins. Med Sci (Paris) 2008 ; 24 : 918-20.

13. Proudhon C, Bourc'his D. Évolution de l'empreinte parentale chez les mammifères : quelle ménagerie ! Med Sci (Paris) $2010 ; 26: 497-503$. 